\title{
PARENTS KNOWLEDGE ABOUT THE DEVELOPMENT OF CHILDREN AGED 2 TO 6 YEARS OLD
}

\author{
Maria da Luz Vale-Dias \\ Institute of Cognitive Psychology, Human and Social Development \\ Faculty of Psychology and Educational Sciences \\ University of Coimbra, Portugal \\ valedias@fpce.uc.pt \\ Luiza Nobre-Lima \\ Research Unit of the Cognitive-Behavioural Research and Intervention Centre \\ Faculty of Psychology and Educational Sciences \\ University of Coimbra, Portugal \\ luizabelima@fpce.uc.pt
}

Fecha de Recepción: 3 Marzo 2018

Fecha de Admisión: 10 Abril 2018

\begin{abstract}
Based on the assumption that parental knowledge about child development influences the way parents understand the behavior of their children, parenting actions and child development, this study aimed to: 1) analyze what parents know about the development of children aged between 2 and 6 years old and the security they have on this knowledge; 2 ) explore the variability of this knowledge according to characteristics such as age, sex, parents' level of education and number of children; 3) know if parental knowledge about child development predicts pleasure in parenting. For the purpose, it was used a socio-demographic questionnaire and the Portuguese versions of the Knowledge of Infant Development Inventory-P [KIDI-P] (MacPhee, 1996; Portuguese adaptation by Nobre-Lima, Vale-Dias, Mendes, Mónico, \& MacPhee, 2014) and of the Pleasure in Parenting Scale [PPS] (Fagot, 1995; Portuguese adaptation: Taborda, Vale-Dias, Aguiar, \& Morais, 2011). KIDI-P is a one-dimensional scale of 58 items which are scored as correct, incorrect or not sure. Three summary scores can be calculated: attempted, accuracy and a total. The former is related to confidence in one s knowledge, the second represents exposure to solid, normative information about infants and the last reflects the percentage of correct answers out of all items on the KIDI-P. PPS is a 10 items unidimensional scale evaluating the gratification in parenting. The sample has around 500 parents, on average on their thirties, both sexes, and not couples. Results show that parental knowledge about child development predicts pleasure in parenting. Results also point to some interesting differences in the KIDI-P summary scores between mothers and fathers and according to the num-
\end{abstract}


ber of children and educational background, that can be suggestive of the importance of addressing the sources of knowledge about developmental information and its rigorous disclosure when working with parents, for example in educational or pediatric contexts.

Keywords: child development; parental pleasure; parental knowledge; knowledge scores; KIDIP; PPS

\section{INTRODUCTION}

Literature is consistent concerning the importance of the knowledge of childrearing and child development to parenthood and children's development and well-being. Several studies have already shown that parental knowledge about child development is a relevant factor capable of influencing the way parents understand the behaviour of their children, parenting actions and ultimately, child development (eg., Bornstein, Cote, Haynes, Hahn, \& Park, 2010; Bornstein, Hahn, \& Haynes, 2011; Goodnow, 1988; Goodnow, \& Collins, 1990; Jahromi, Guimond, Umaña-Taylor, Updegraff, \& Toomey, 2014). Some authors assume that parent beliefs are cognitions involved in dynamic belief systems model (Sigel, \& McGillicuddy-DeLisi, 2002), showing an important role in parenting. In fact, authors assume that "parents' cognitions, particularly their knowledge about children's development, are an important dimension of parenting competence" (Jahromi et al., 2014, 593). It seems that the impact of that knowledge is important to both the parents' beliefs and practices and the child development: "parenting knowledge helps form parents' beliefs and practices with their children and, in turn, children whose parents have more accurate knowledge about such things as developmental milestones and effective childrearing practices show better cognitive and behavioral outcomes (Benasich, \& BrooksGunn, 1996; Dichtelmiller et al., 1992; Whitman, Borkowski, Keogh, \& Weed, 2001)" (Jahromi et al., 2014, 593). Thus, further research on parents' knowledge about development can inform about the origins and variability of parental beliefs, making of these an important field of intervention.

Also, the relationship of parenting and psychological well-being has been an important target of research in the last decades (e.g., Glass, Simon, \& Andersson, 2016; Hansen, 2012; Nelson, Kushlev, English, Dunn, \& Lyubomirsky, 2013), with studies showing controversial results (Bhargava, Kassam, \& Loewenstein, 2014). On its examination, attention have been paid to the pleasure that parents feel when they carry out routine caretaking acts with their children. Previous literature indicates that the activities of parental interaction with their children and the parents' gratification associated with this interaction constitute an indispensable daily requisite for a consistent adaptation of the child (e.g., Chandani, Prince \& Scott, 1999; Fagot, 1995; Nabuco, Aguiar, Costa, \& Morais, 2014; Taborda, Vale-Dias, Aguiar \& Morais, 2011; Tamis-Lemonda, Shannon, Cabrera, \& Lamb, 2004). Given that knowledge about child development seems to influence parental practices, and its outcomes and success, we may assume that it also influences pleasure that parents feel in parenting actions. Studies on this issue are needed in the international and Portuguese contexts.

Based on the assumption that parental knowledge about child development is an important field of research, relevant to parenting actions (Bornstein et al., 2010) and with rare works in Portugal (see Nobre-Lima, Vale-Dias, Mendes, Mónico, \& MacPhee, 2014), the present study has three main goals. First, it aims to analyse what parents know about the development of children aged between 2 and 6 years old, and the security they have on this knowledge. Secondly, it explores the variability of this knowledge according to characteristics such as age, sex, level of education or educational background and number of children, also examining if these aspects are associated to pleasure in parenting. Finally, it aims to know if parental knowledge about child development predicts pleasure in parenting. 


\section{METHOD}

\section{Participants}

Participants were 540 parents (mothers and fathers) of children aged between 2 and 6 years old, who agreed to collaborate. Mother-father dyads were not included in this study. The vast majority of subjects were Portuguese (96,9\%). The sample (Table 1) comprised 62,8\% mothers and $32,2 \%$ fathers, aged between 21 and 55 years old $(M=35,79, S D=5,26)$. For the present study two age groups were considered: Group 1, that included subjects aged between 21 and 36 years old $(21<$ Group1<36); and Group 2, including participants between 37 and 55 years old $(37<$ Group $2<55)$. The distribution of subjects by marital status revealed that $75,2 \%$ of them were married, $14,4 \%$ lived in non-marital partnership, $4,8 \%$ were single and $4,6 \%$ divorced. Referring to the number of children, $46,9 \%$ had a single child, $44,1 \%$ had two children and the rest $9,1 \%$ had 3 , 4 or 5 children. Concerning to the participants' educational background, $22,2 \%, 32,4 \%$ and $45,4 \%$ had respectively primary, secondary and higher education. In what respects to the socio-economic level, $50,9 \%$ were from a medium level, $28,1 \%$ were from a low level and 19,8\% from a high level. Only three parents were unemployed $(0,6 \%)$.

Table 1.

Sample Description ( $N=540$ parents)

\begin{tabular}{|c|c|}
\hline Sociodemographic Characteristics & $\%$ \\
\hline \multicolumn{2}{|l|}{ Gender } \\
\hline Male & $32,2 \%$ \\
\hline Female & $62,8 \%$ \\
\hline TOTAL & $100 \%$ \\
\hline \multicolumn{2}{|l|}{ Marital status } \\
\hline married & $75,2 \%$ \\
\hline non-marital partnership & $14,4 \%$ \\
\hline single & $4,8 \%$ \\
\hline divorced & $4.6 \%$ \\
\hline TOTAL & $100 \%$ \\
\hline \multicolumn{2}{|l|}{ Educational background } \\
\hline primary & $22,2 \%$ \\
\hline secondary & $32,4 \%$ \\
\hline higher education & $45,4 \%$ \\
\hline TOTAL & $100 \%$ \\
\hline \multicolumn{2}{|l|}{ Socioeconomic Status } \\
\hline Low & $28,1 \%$ \\
\hline Medium & $50,9 \%$ \\
\hline High & $19,8 \%$ \\
\hline TOTAL & $100 \%$ \\
\hline \multicolumn{2}{|l|}{ Number of children } \\
\hline 1 & $46,9 \%$ \\
\hline 2 & $44,1 \%$ \\
\hline 3,4 or 5 & $9,1 \%$ \\
\hline TOTAL & $100 \%$ \\
\hline
\end{tabular}

\section{Measures}

Data collection was made through an assessment protocol that included a socio-demographic questionnaire and the Portuguese versions of the Knowledge of Infant Development Inventory-P [KIDI-P], originally developed by MacPhee (2002), and of the Pleasure in Parenting Scale [PPS] (Fagot, 1995). The participation was voluntary and the answers were anonymous. 
The Socio-demographic questionnaire was used to collect information about parents and their parental characteristics.

The Portuguese version of the Knowledge of Infant Development Inventory-P [KIDI-P] (MacPhee, 2002; Portuguese adaptation: Nobre-Lima, Vale-Dias, Mendes, Mónico \& MacPhee, 2014 ) is a self-report instrument formed by a unidimensional scale of 58 items which are scored as correct, incorrect or not sure. The KIDI-P delivers comprehensive information about early development and was created to assess a person's factual knowledge of parental practices, child developmental processes and norms of child behaviour in the age range from 2 to 6 years old (MacPhee, 2002). This inventory allows for the calculation of three summary scores (Attempted, Accuracy and Total) that reflect correctness and confidence in one's knowledge. The Attempted Index is related to confidence in one s knowledge (nonuse of the Not Sure option); the Accuracy Index represents exposure to solid, normative information about infants (answering items correctly); and the Total Index reflects the percentage of correct answers out of all items on the KIDI-P. The internal consistency (Cronbach's alpha) of the Portuguese version is high (.89), and is slightly higher than the original version's alpha (Nobre-Lima et al., 2014).

The Pleasure in Parenting Scale [PPS] (Fagot, 1995; Portuguese adaptation: Taborda, ValeDias, Aguiar, \& Morais, 2011) is a unidimensional scale composed by 10 items that describe different tasks of daily care and/or interaction between parents and children (e.g., putting the child to bed, bathing the child, etc.) and aims to evaluate, on a 5 points Lickert scale, the gratification of the parents when they perform care/support tasks with their child. In the original version, the scale presented coefficients of Cronbach's alpha of .81 for mothers and .76 for fathers. The Portuguese version presented a Cronbach's alpha of .75 (mothers and fathers).

\section{RESULTS}

\section{Parental pleasure, correctness and confidence in one's knowledge: Descriptive Statistics}

The average scores show important values of gratification (PPS), revealing that parents refer high pleasure in parenting actions (Table 2).

In terms of average scores on the KIDI-P indexes (Table 2), the mean for the Total Index was $.64(\mathrm{SD}=.091)$ reflecting a fair mastery of developmental knowledge. The Attempted and Accuracy Indexes were higher, respectively $.88(\mathrm{SD}=.108)$ and $.74(\mathrm{SD}=.073)$. Significant differences exist between them, meaning that parents are, generally, more confident than accurate or precise on their knowledge $(\mathrm{t}(539)=22.464, \mathrm{p}<.000)$.

Table 2.

Descriptive Statistics (scores of PPS and KIDI-P)

\begin{tabular}{llllll}
\hline & $\mathrm{N}$ & Minimum & Maximum & Mean & Std. Deviation \\
\hline Total PPS & 540 & 28.00 & 50.00 & 41.4352 & 4.46731 \\
\hline Attempted_KIDI-P & 540 & .40 & 1.00 & .8763 & .10823 \\
\hline Accuracy_KIDI-P & 540 & .41 & .95 & .7363 & .07307 \\
\hline Total_KIDI-P & 540 & .31 & .86 & .6433 & .09077
\end{tabular}

PPS - Pleasure in Parenting Scale; KIDI-P - Knowledge of Infant Development Inventory-P 
Analyses of parent differences on Knowledge about child development and Pleasure in parenting: sex, age, educational background, number of children

In what concerns to $\mathrm{KIDI}-\mathrm{P}$, mothers $(\mathrm{M}=.742, \mathrm{SD}=.070)$ are more accurate than fathers $(\mathrm{M}=.727, \mathrm{SD}=.077)$ on their knowledge about development $(\mathrm{t}(538)=-2.261, \mathrm{p}<.03)$, as they $(\mathrm{M}=.653$, $\mathrm{SD}=.087)$ show to know more than fathers $(\mathrm{M}=.628, \mathrm{SD}=.096)$ about development of pre-school children $(t(538)=-3.077), p<.003)$. However, there are no differences between fathers and mothers on PPS- Pleasure in parenting $(\mathrm{t}(538)=.11, \mathrm{p}=.74)$.

None of the KIDI-P Indexes vary when considering age groups (Group 1: 21 to 36 years old; and Group 2: 37 to 55 years old). Also, regarding to Pleasure in parenting, age showed only low negative correlations with the score of PPS $(r=-.136 ; p<.001)$.

Related to differences by Educational background, there are only differences in the Accuracy Index $(F(2,537)=12,244, p<.000)$. Tukey HSD post-hoc analyses revealed that parents with primary education $(\mathrm{M}=.711, \mathrm{SD}=.074)$ are less accurate on their knowledge about development than parents with secondary education $(\mathrm{M}=.735, \mathrm{SD}=.070 ; \mathrm{p}<.02)$ or with higher education $(\mathrm{M}=.750, \mathrm{SD}=.072$; $\mathrm{p}<.000)$. Yet, there are no differences in Pleasure in parenting (PPS) by Educational background ( $\mathrm{F}=$ $1,354, p=.24)$.

When comparing parents with only one child (G1) with those that have two or more (G2), Attempted and Total Indexes are higher for the second group. (respectively, $\mathrm{G} 1: \mathrm{M}=.870, \mathrm{SD}=.109$; $\mathrm{G} 2: \mathrm{M}=.890, \mathrm{SD}=.106 ; \mathrm{t}(538)=-3.123, \mathrm{p}<.003$ and $\mathrm{G} 2: \mathrm{M}=.633, \mathrm{SD}=.090 ; \mathrm{G} 2: \mathrm{M}=.652, \mathrm{SD}=.091$; $t(538)=-2.480, p<. .02)$. This means that parents with more children tend to know more about child development and be more confident about what they know.

\section{Parental knowledge about child development and prediction of pleasure in parenting}

Linear regression analysis showed that parental knowledge about child development predicts pleasure in parenting. The standardized Coefficient (Beta $=.114 ; p=.008$ ) suggests a positive relationship.

\section{DISCUSSION AND CONCLUSIONS}

According to data displayed, parents are fairly knowledgeable and secure about development of pre-school aged children, revealing that they are exposed to solid information about child development. They also present very good levels of pleasure in parenting actions, corroborating some data of those studies that refer to the positive aspects of parenting (Nelson et al., 2013).

Results showing differences between mothers and fathers in terms of knowledge about child development are somewhat convergent with previous studies (Schreiber, 2001). Yet, indexes of KIDI-P didn't show any relation with parents' age. However, they varied according to the number of children those parents have, which might stress the role of experience and relationship to the awareness that parents have about children, their behaviour and their development.

As in this study, the influence of educational background on the accuracy of knowledge about child development has already been stated (Bornstein et al., 2010), thus valuing parents' personal resources as important factors to the understanding of their children. On the other hand, there are no differences by educational background in pleasure in parenting, revealing that access to different levels of education does not imply access to differential mechanisms in terms of the perception of pleasure in parenting activities. Age and sex also revealed no relevant association to pleasure in parenting activities, suggesting that pleasure in parenting does not seem to be associated with conceptions around male and female roles or due to an age profile of the parents.

Globally, these findings reinforce the relevance of research on parental believes about their children development in the $21^{\text {st }}$ century. Regardless the amount of easily accessible information that 
exists nowadays in industrialized societies, it is still important to identify the factors that contribute to have better informed parents about the characteristics that are expected to emerge in a particular stage of development. This fact becomes even more relevant if we take into account that, according to our data, parental knowledge about child development is a predictor of pleasure in parenting activities.

Although this research should be conducted with other and larger samples of parents, preferably in a cross-cultural approach, it allows to suggest the need of investing on a comprehensive analysis of the abovementioned variables. It is still important to identify the factors that contribute to pleasure in parenting and to have better informed parents about the characteristics that are expected to arise in a specific stage of development.

\section{REFERENCES:}

Bhargava, S., Kassam, K.S., \& Loewenstein, G. (2014). A reassessment of the defense of parenthood. Psychol Sci., 25(1), 299-302. DOI: 10.1177/0956797613503348

Bornstein, M. H., Cote, L. R., Haynes, O., Hahn, C., \& Park, Y. (2010). Parenting knowledge: Experiential and sociodemographic factors in European American mothers of young children. Developmental Psychology, 46, 1677-1693. D0I:10.1037/a0020677

Bornstein, M. H., Hahn, C.-S., \& Haynes, 0. M. (2011). Maternal Personality, Parenting Cognitions and Parenting Practices. Dev. Psychol., 47(3), 658-675. D0I:10.1037/a0023181

Chandani, K., Prince, M. \& Scott, S. (1999). Development and initial validation of the parent-child joint activity scale: a measure of joint engagement in activities between parent and pre-school child. International Journal of Methods in Psychiatric Research, 8 (4), 219-228. D0I: $10.1002 / \mathrm{mpr} .73$

Fagot, B. (1995). Development of pleasure in parenting scale. Early development and parenting, 4 (2), 75-82. DOI: 10.1002/edp.2430040204

Glass, J., Simon, R.W., \& Andersson, M.A. (2016). Parenthood and Happiness: Effects of WorkFamily Reconciliation Policies in 22 OECD Countries. AJS, 122(3), 886-929. DOI: 10.1086/688892

Goodnow, J. J. (1988). Parents' ideas, actions and feelings: Models and methods for developmental and social psychology. Child Development, 59, 286-320. D0I:10.2307/1130312

Goodnow, J. J., \& Collins, W. A. (1990). Development according to parents. The nature, sources, and consequences of parents' ideas. Hillsdale, NJ: Erlbaum.

Hansen, T. (2012). Parenthood and Happiness: A Review of Folk Theories Versus Empirical Evidence. Social Indicators Research, 108, 29-64. D0I: 10.1007/s11205-011-9865-y

Jahromi, L. B., Guimond, A. B., Umaña-Taylor, A. J., Updegraff, K. A., \& Toomey, R. B. (2014). Family Context, Mexican-Origin Adolescent Mothers' Parenting Knowledge, and Children's Subsequent Developmental Outcomes. Child Development, 85(2), 593-609. D0I:10.1111/cdev.12160

MacPhee, D. (2002). Manual: Knowledge of infant development inventory. Unpublished manuscript, Colorado State University, Fort Collins, CO.

Nabuco, M. E., Aguiar, M. S., Costa, C., \& Morais, D. (2014) Evaluation of the effectiveness of the implementation of the A PAR parental intervention programme in Portugal. Child development and parenting support. European Early Childhood Education Research Journal, 22(4), 554-572. DOI: $10.1080 / 1350293 X .2014 .947836$

Nelson, S. K., Kushlev, K., English, T., Dunn, E. W., \& Lyubomirsky, S. (2013). In defense of parenthood: Children are associated with more joy than misery. Psychological Science, 24, 3-10. DOI: $10.1177 / 0956797612447798$ 
Nobre-Lima, L., Vale-Dias, M. L., Mendes, T., Mónico, L., \& MacPhee, D. (2014): The Portuguese version of the Knowledge of Infant Development Inventory-P (KIDI-P), European Journal of Developmental Psychology, 11(6), 740-745. DOI: 10.1080/17405629.2014.929941

Taborda, J., Vale-Dias, M. L., Aguiar, M. S. \& Morais, D. (2011). Presentation of the portuguese version of two different scales: Pleasure in Parenting Scale - PPS (Escala de Gratificação Parental, EGP) and Parent-Child Joint Activity Scale - PJAS (Escala de Actividades de Interacção PaisFilhos, EAIP/F). In E. Catarsi et J.-P. Pourtois (Eds.), Education familiale et services pour l'enfance, II vol. (pp. 264-268). Firenze: Firenze University Press.

Tamis-Lemonda, C., Shannon, J., Cabrera, N. \& Lamb, M. (2004). Fathers and mothers at play with their 2- and 3- years olds: Contributions to language and cognitive development. Child Development, 75, 1806-1820. D0I: 10.1111/j.1467-8624.2004.00818.x

Schreiber, M. A. (2001). Confirmatory factor structure of a measure of knowledge of child development (Unpublished Master's thesis). Middle Tenesse State University, Murfreesboro, TN.

Sigel, I. E., \& McGillicuddy-DeLisi, A. V. (2002). Parent beliefs are cognitions: The dynamic belief systems model. In M. H. Bornstein (Ed.), Handbook of parenting: Being and becoming a parent (pp. 485-508). Mahwah, NJ: Erlbaum. 
